\title{
EFFECTIVENESS OF VESTIBULAR STIMULATION IN CHILDREN WITH DEVELOPMENTAL COORDINATION DISORDER
}

\author{
GANAPATHY SANKAR $\mathbf{U}^{1}$, MONISHA $\mathbf{R}^{1 *}$, CHRISTOPHER AMALRAJ VALLABA DOSS ${ }^{2}$, PALANIVEL RM ${ }^{3}$
}

${ }^{1}$ Ph.D Scholar, SRM College of Occupational Therapy, SRM Institute of Science and Technology, Kattankulatur 603203, India. ${ }^{2} \mathrm{College}$ of Medicine, Imam Abdulrahman Bin Faisal University, Dammam 31441, Saudi Arabia. ${ }^{3}$ Deanship of Quality and Academic Accreditation, Imam Abdulrahman Bin Faisal University, Dammam 31441, Saudi Arabia. Email: dreamsfuture000@gmail.com

Received: 19 November 2019, Revised and Accepted: 30 December 2019

ABSTRACT

Objectives: The objectives of this study were to find out the effects of vestibular stimulation in developmental coordination disorder (DCD) children.

Methodology: Thirty children $(n=30)$ were screened using the DCD questionnaire (DCDQ). After baseline screening among the 30 children, 15 were confirmed as suspects of DCD. Vestibular stimulation was given for all the 15 children.

Results: It is revealed that the motor coordination was improved significantly in children with DCD.

Conclusion: The present study concluded that vestibular stimulation is effective to enhance performance in tasks requiring motor coordination.

Keywords: Developmental coordination disorder, Vestibular stimulation, Indian context, Primary school children.

(C) 2020 The Authors. Published by Innovare Academic Sciences Pvt Ltd. This is an open access article under the CC BY license (http://creativecommons. org/licenses/by/4. 0/) DOI: http://dx.doi.org/10.22159/ajpcr.2020.v13i3.36575

\section{INTRODUCTION}

Developmental coordination disorder (DCD) impacts motor activity and affects nearly $6.7 \%$ of Indian children and affects the person's ability to communicate and interact with a peer group. Children with DCD have trouble with academic activities, and communication skills lack participation in physical activities. DCD can be diagnosed based on a gold standard assessment tool but their lies inconsistency among the tools that identify children as DCD [1]. DCD questionnaire (DCDQ) should be administered to screen children suspected to have DCD. These children experience difficulty in performing physical activity and social interactions. Children with DCD remain alone and tend to escape from peer group interaction and always confound at home. Dr. Ganapathy Sankar in his research on evaluation of balance skill in children with DCD, he has analyzed that there is an inability in performing a physical activity like the peer group children of the same age and they have a deficit in motor control and encounter imbalance when exposed to gait initiation in an unfamiliar environment [2].

It has been shown that imbalance leads to the inhibition of gait adaptations which are evident by shorter steps, greater trunk inclination, decreased ankle plantar, and dorsiflexion and concluded that children with DCD physically, psychologically, and socially avoid participating in physical activities to avoid injuries due to their difficulty in balance. Thus, early identification and treatment of balance control difficulties are in increasing need. Children with DCD are often isolated and diverted when engaged with particular activities; they also lack the motivation to play and to participate in academic activities [3]. Few children with DCD often have repetitive movements such as hand flapping and head banging. Hyperresponsiveness to sensory input is due to poor sensory modulation skills and sensory processing skills. Their lays an inconsistency among the assessment tools used to identify these children. Movement assessment battery for children (MABC), Bruininks-oseretsky test of motor proficiency (BOTMP), Developmental coordination disorder questionnaire (DCDQ) some of the tools available for screening, diagnosing or identifying motor coordination difficulty in children [4]. These tools are not relevant to the Indian context due to the influence of multicultural and multilinguistic Indian environments. Therefore, a unique instrument for screening and assessment of individuals with DCD is needed for the Indian population for several reasons.

In turn, tonic postural support reactions of legs are called on to correct body position and avoid loss of balance within a vertical position, occupational therapy intervention includes jumping on the trampoline to overcome this problem. This linear activity uses gravity to provide constant stimuli, promoting the tonic postural support muscular adaptation which is needed for maintaining an upright position when landing on the trampoline [5]. As long as the constant rhythm is sustained in jumping, occupational therapist observes the adaptive response, in which extension of the legs is less while jumping up and the extension of legs is more while jumping down. If there is an offbalance movement, which often occurs when jumping on a trampoline, there will be a transient break in the velocity and the rhythm and the direction of the head movement will be occurred, which, in turn, causes equilibrium reaction to prevent falling $[6,7]$.

Various studies found that Arnold, 2008; Smith and Bryan, 1999; and Bonadonna, 1989, vestibular stimulation reduces hypersensitivity in DCD, in which there is no study to determine the effectiveness of vestibular stimulation to reduce coordination difficulty in DCD [8]. Hence, this research has been carried out to determine the effectiveness of vestibular stimulation in DCD.

\section{METHODOLOGY}

After the proposal presentation to the Ethics Committee from SRM College of Occupational Therapy, procedure clearance was obtained and the children who were diagnosed as DCD were selected for the study. Samples were collected from private therapy centers in and around Chennai using a convenient sampling technique. Children who were with a pervasive developmental disorder and any other physical and mental impairment were excluded out from the study. Consent forms were obtained from the child's parents or caregivers and the study was clearly explained to them. Thirty $(n=30)$ participants were recruited for the study. Pre-test scores were obtained on the $1^{\text {st }}$ day of the data collection period and intervention was carried out. Children received vestibular stimulation for 3 days in a week for 2 months. Post- 
test scores were obtained after the intervention period. The vestibular stimulation program provided 1 min of rocking in a linear direction in each of the following positions:

- Prone on therapy ball: Movement was provided in anterior-posterior, lateral, and up and downward directions

- Supine on therapy ball: Movement was provided in anterior-posterior, lateral, and up and downward directions

- Seated on a tilt board: Movement was provided in anterior-posterior, lateral, and up and downward directions.

\section{RESULTS}

Paired " $t$ "-test was used to find out the effectiveness of vestibular stimulation on the severity of DCD and the results revealed that there was a statistically significant difference $(" \mathrm{t} "=10.566, \mathrm{p}<0.05)$ after vestibular stimulation among children with DCD (Table 1).

\section{DISCUSSION}

The present study was carried out to find out the effectiveness of vestibular stimulation in reducing the severity of motor coordination while performing selected activities among DCD children [6]. Thirty children who were diagnosed as DCD were randomly selected for the study. DCDQ was used to analyze whether children were suspect of DCD. Vestibular stimulation was delivered for 3 days a week for 2 months. To avoid social isolation and to enhance social aptitudes, few centers based on intercessions are suggested. Social abilities can be prepared in gathering and, furthermore, in clinical setups, Picture Exchange Communication System to enhance social correspondence, joint consideration preparing to enhance joint consideration, activity-based mediations to enhance social aptitudes, computer-based mediations to enhance social aptitudes, naturalistic behavioral mediations to enhance social correspondence, developmental mediations to enhance social correspondence, parent-interceded mediations, and impersonation preparing to enhance social correspondence, behavioral methods to enhance limited and tedious behaviors, physical action to diminish limited and tedious behaviors, recess mediation, relaxation gathering, and social stories to enhance recreation cooperation, use of favored, centered, or confined uncommon interests to enhance social behaviors, play expertise intercessions to enhance play execution, social stories, and associate interceded mediations to enhance social abilities.

To understand the relationship between the bodies to space by the brain, the gravity of the movement should interact with the muscles and joints through proprioceptive sensations and verification of the visual system is also needed to confirm what is being sensed. In children with DCD, all these senses are not modulated and synchronized properly. Furthermore, certain activities seem preservative such as spinning or toe walking, may actually be that brain's way of trying to stabilize the body, seeking vestibular stimulation of semicircular canals angular movements that are fast in space should be provided to develop the compensatory body posture and tonic reflexes. It may be the brain's functional sensory adaptation based on the feedback information. Occupational therapy often treats vestibular issues by placing the child in a suspended hammock and rotating to stimulate the semicircular canals to accommodate new vestibular feedbacks [4-6]. The physical act of jumping stimulates the utricles in the inner ear which detects the linear motion and head is tilted. The utricle is in a horizontal plane when the head is upright. Hair cells in

Table 1: Comparison of pre-test scores and post-test scores of vestibular stimulation

\begin{tabular}{lllll}
\hline Test & Mean & SD & "t"-test & "p"-value \\
\hline Pre-test & 164.20 & 6.428 & 10.566 & $0.001^{*}$ \\
Post-test & 134.40 & 7.854 & & \\
\hline
\end{tabular}

Level of significance: $\mathrm{p}<0.05$. *Significant $<0.05$ in the effectiveness of vestibular stimulation on the severity of DCD. DCD: Developmental coordination disorder, SD: Standard deviation every four quadrants of the utricle are systematically oriented in different directions. As the head moves, gravitational and linear movement forces act on the otoliths, which stimulate hair cells accordingly to indicate the linear or tilted position of the head.

Vestibular stimulation is familiarized as a new rehabilitative strategy which contributes to solving various issues of visual perceptual defects, motor incoordination, cognitive, and emotional process. The present study suggests vestibular stimulation for children with DCD as it increases cognition, and it is considered to be the most powerful treatment strategy. However, it is a non-invasive form of treatment and considered both safe and effective. The vestibular system functions effectively to maintain the nervous system in a balanced state. Vestibular stimulation practiced in children with DCD calms down the information received by utricle and stimulates it for information processing [8-10]. However, fast vestibular stimulation activates the semicircular canal and processes the information. Arnold et al., in 1985 , carried out a similar study on vestibular stimulation and visual stimulation as a treatment. Thirty children were recruited for the study and all received combined eyes opened vestibular stimulation. The vestibular alone condition showed a greater effect when compared to the control condition. Bhatara et al., in 1981, found that vestibular stimulation in rotational movement stimulates the semicircular canals, which, in turn, reduces the behavior problem. After receiving vestibular stimulation, the sensory processing issues are reduced, which, in turn, increase the performance level for children with DCD [8]. The results stated that there was a change in the learning and behavior of DCD children. It also states that the vestibular and tactile systems have more influence on the processing of input over sensory channels.

\section{CONCLUSION}

This study determined the effectiveness of vestibular stimulation in reducing hypersensitivity and enhances the performance of DCD children. Vestibular stimulation reduces the sensory processing issues in children with DCD, which, in turn, the behavioral issues are also reduced and this has an impact on the severity of DCD, so vestibular stimulation can be used as one of the therapeutic interventions.

\section{Future recommendations}

This present study can be carried out in a large sample size with longer time duration for intervention to know the long-term impact on the intervention. This study can also be carried out to determine the effects of vestibular stimulation in learning and in behavioral changes.

\section{ACKNOWLEDGMENTS}

The authors would like to thank the chancellor of SRM Institute of Science and Technology and also thank to all the children and parents who participated in the study. Last but not least, I thank all of them whose names have inadvertently failed my memory and who in their own unique way has made this project a reality.

\section{AUTHORS' CONTRIBUTIONS}

Ganapathy Sankar participated in the design of the study and performed the statistical analysis and Monisha. $\mathrm{R}$ contributed to draft the manuscript, participated in the design of the study and the gathering of data, and helped to draft the final manuscript.

\section{SOURCE OF FUNDING}

Self.

\section{CONFLICTS OF INTEREST}

Nil.

\section{REFERENCES}

1. Sankar UG, Monisha R. Evaluation of cardio-vascular risk in children with developmental coordination disorder in Indian context-pilot study. 
Res J Pharm Tech 2018:11:5405-7.

2. Sankar UG, Saritha S. A study of prevalence of developmental coordination disorder (DCD) at Kattankulathur, Chennai. Indian J Physiother Occup Ther 2011;5:63-5.

3. Sankar G, Monisha R. Life impact of developmental coordination disorder: Qualitative analysis of patient and therapist experiences. Biomed Pharmacol J 2019;12:491-4.

4. Shankar UG, Monisha R. Assessment of balance in children with developmental coordination disorder in Indian context. Indian J Public Health Res Dev 2019;10:67-70.

5. American Psychiatric Association. Diagnostic and Statistical Manual of Mental Disorders. $5^{\text {th }}$ ed. Arlington, VA: American Psychiatric Association; 2013

6. Monisha R, Manikumar M, Krishnakumar A. Evaluating the effectiveness of phonophoresis by piroxicam and dimethyl sulfoxide for women's with osteoarthritis knee joint. Asian J Pharm Clin Res 2018;11:329-31.

7. Senthilselvi R, Boopana M, Sathyan L, Visuvasam P, Ganesan V. Drug utilization pattern in pediatric patients in a secondary care hospital. Int J Pharm Pharm Sci 2019;11:69-74.

8. Thenmozhi P, Priya P. Factors contributing for noncompliance of follow-up care among post cardiac patients. Int J Pharm Pharm Sci 2019;11:22-5.

9. Sharma S, Seth N. Optimization of component variables by statistical approach for design of chronotherapeutic dosage form for hypertension. Asian J Pharm Clin Res 2019;12:64-72.

10. Horowitz B, Woods KR. Development of hemagglutination assays I. Attachment of anti-HBs antibody to stabilized erythrocytes. Vox Sang $1977 ; 33: 324-34$ 